\title{
The cultural aspects of the translation of the Yakut heroic epic Olonkho to French
}

\author{
[Les aspects culturels de la traduction de l'epopee heroique yakoute \\ Olonkho vers le francais]
}

\author{
Liudmila Zamorshchikova - Marianna Samsonova - Lilya Spektor - Irena \\ Khokholova
}

DOI: 10.18355/XL.2019.12.01XL.07

\begin{abstract}
This article deals with the problems of translation and interpretation of the text of the Yakut heroic epos Olonkho into French and reveals and explains the solutions made by the translator. The difficulties of the first order which make obstacles for the translator are the realia and the notions charged with the specific connotations of the culture of the original text. The various possibilities of translation and transfer of cultural peculiarities linked to ethnic concepts and concepts are shown in the articles: the translation processes aiming to preserve the specific image such as transliteration, and on the other hand, the processes which " naturalize "objects of culture by means of the adaptation of foreign concepts for the receiving culture.
\end{abstract}

Key words: Yakut language, heroic epos, Olonkho, realia, translation, transfer of cultural concepts, cultural references

\section{Résumé}

Le présent article aborde des problèmes de traduction et d'interprétation du texte de l'épopéé yakoute vers le français et révèle et explique les solutions réalisées par le traducteur. Les difficultés du premier ordre qui font des obstacles pour le traducteur sont les realia et les notions chargées des connotations spécifiques propres à la culture $\mathrm{du}$ texte original. Les diverses possibilités de traduction et de transferts des particularités culturelles liées avec les notions et concepts ethniques sont montrées dans l'articles : les procédés de traduction visant à conserver l'image spécifique telle que translittération, et d'autre part, les procédés qui «naturalisent» les objets de culture à l'aide de l'adaptation des concepts étrangers pour la culture réceptrice.

Les mots-clés : langue yakoute, épopée héroïque, Olonkho, realia, traduction, transfert des concepts culturels, références culturelles

\section{Introduction}

Comme remarque G. Mounin, on peut admettre que «...l'existence de cultures ou de civilisations différentes, constituant autant de mondes bien distincts, est une réalité démontrée. On peut admettre aussi que, dans une mesure qui reste à déterminer, ces mondes distincts sont impénétrables les uns pour les autres. Et ces hiatus entre deux cultures données s'ajoutent aux difficultés que les langues ellesmêmes opposent à la traduction totale» (G. Mounin, 1980). La traduction et l'interprétation sont des activités humaines consacrées à la résolution de l'ensemble des problèmes linguistiques et culturels, puisque chaque langue se distingue de l'autre par sa manière de classifier la réalité objective. Le problème de l'interprétation du texte se révèle fondamental pour la traduction du texte littéraire. «L'interprétation du texte est un processus inférenciel qui consiste à construire des hypothèses à partir de certaines prémisses qui sont, d'une part, fournies par le texte et, d'autre part, extraites par le lecteur de l'ensemble de ses connaissances (croyances, opinions) générales. Comme tout autre lecteur, le traducteur construit ses propres hypothèses concernant, 
d'une part, le monde du texte et, d'autre part, l'attitude de l'auteur vis-à-vis de ce monde » (Sv.Vogeleer, 1995).

Selon E. Tarassov, la cause essentielle de malentendu et de l'incompréhension en communication interculturelle ne consiste pas en différences des langues, mais en différences des consciences nationales (ethniques) des locuteurs (communicants). A cette clarté, le dialogue des cultures différentes ne se présente autant que la communication des consciences différentes, la communication des images des cultures différentes à l'intérieur des consciences des personnes communicantes. (Tarassov, 1996). Dans les processus mentaux de cognition les objets de la culture se découvrent au lecteur par ses aspects différents. «Les qualités des objets de la culture se qualifient comme naturelles, fonctionnelles et systémiques ». La compréhension des qualités naturelles et fonctionnelles des objets des cultures proches ne présente pas de difficultés. C'est une autre affaire quand il s'agit de compréhension des qualités systémiques intégrales qui ne surgissent et ne se révèlent qu'en corrélation avec des systèmes où elles fonctionnent (Tarassov, 1998). Leur caractère symbolique s'ouvre au lecteur qui possède les connaissances du système dans lequel un objet de la culture acquiert ces qualités. On peut considérer comme de pareilles difficultés la traduction des références culturelles et realia qui sont liés aux associations et connotations ethniques se rapportant aux traditions historiques et culturelles, à la spécificité de la «vision du monde».

\section{Olonkho - le sommet du folklore yakoute}

Olonkho du peuple yakoute ou sakha (l'autodénomination du peuple) est l'une des dernières traditions orales vivantes des peuples de Sibérie. Olonkho c'est le nom qui désigne à la fois la tradition des épopées héroïques ainsi que les oeuvres concrètes. L'histoire d'olonkho compte selon les différentes sources plus de 1200 ans (Ivanov, 2016). En 2005 l'Olonkho yakoute a été porté par la décision de l'UNESCO dans la liste des chefs d'oeuvre du patrimoine immatériel de l'humanité. L'olonkho se compose en moyenne de 10 000-15000 lignes, mais il y a des grands olonkhos comptant 40 000-56 000 lignes. Un seul interprète, chanteur-conteur - olonkhossoute, retenant par coeur les miliers de vers, alterne une partie versifiée chantée avec une partie en prose composée de récitatifs. Les paroles des héros principaux doivent être chantées avec la basse, les parties des jeunes héros sont chantées avec le ténor, les forts du monde inférieur sont chantées avec une voix cassée et de manière injuste... Le conteur imite le henissement des chevaux, le chant des oiseaux, les cris des animaux immergeant le public dans le monde fabuleux d'olonkho.

Comme toute œuvre orale, l'olonkho remonte à la culture de la pré-écriture, lorsque l'épopée était une priorité dans la vie spirituelle du peuple. Olonkho s'est développé partout, spontanément, en accumulant et en reproduisant des valeurs spirituelles, des idées de base sur le monde environnant et, en général, sur l'expérience historique et sociale du peuple. Dans chaque village il y avait environ une douzaine d'olonkhossoutes. Dans les années 40 du XXème siècle dans les 13 régions de la république 83 olonkhosuts ont enregistré 396 scènes d'olonkho, ce qui témoigne de l'existence généralisée d'olonkho dans le passé. Des traditions épiques locales ont été formées dans chaque région de Yakoutie. L'épopée héroïque yakoute - olonkho a

XLinguae, Volume 12 Issue 1XL, January 2019, ISSN 1337-8384, eISSN 2453-711X 
toujours été au centre de l'attention des chercheurs d'histoire, de culture et d'héritage spirituel du peuple sakha.

Les sujets d'olonkho peuvent être divisés en trois groupes. Le premier groupe réunit les olonkhos parlant de la création de la Terre ou du Monde du Milieu par les habitants du Monde Supérieur - les bonnes divinités. Le deuxième sujet fréquent raconte la façon dont les exilés du Monde Supérieur dirigés par Er-Sogotokh (Le Solitaire) ont donné naissance au peuple yakoute. Le troisième sujet - le héros du Monde du Milieu Niourgoun Bootour, rivalise avec des démons diaboliques abaa'hy $\mathrm{du}$ Monde Inférieur qui luttent pour exterminer les humains ou au moins ruiner leurs vies. Les légendes reflètent les souvenirs du passé lointain du peuple, décrivent le pays ensoleillé où il n'y a jamais d'hiver, où vivaient les ancêtres des Yakoutes avant de se déplacer sur les rives de la Lena et du Vitim, dans la froide taïga. On trouve dans l'olonkho la cosmogonie des croyances yakoutes, les valeurs familiales de la société qui venait de passer dans le patriarcat et conservait encore des éléments du matriarcat, les combats avec les forces maléfiques reflétant les guerres avec les voisins hostiles... L'un des principaux traits de l'olonkho en tant que genre est son historicisme particulier - l'olonkho est conçu, créé et sert d'histoire particulière de la société humaine dans son ensemble (comme c'est souvent le cas dans les contes épiques), d'histoire pas réelle, mais mythologique.

Les premières fixations écrites d'olonkho datent des années 1840. L'académicien russe Alexandre Middendorf lors de son voyage en Sibérie Orientale a noté le début d'olonkho d'un interprète inconnu, le résumé de ce texte en russe a été publié dans le recueil "Les échantillons de la littérature populiare yakoute » en 1911. Depuis 1840 plusieurs chercheurs russes et yakoutes ont étudié et édité les textes originaux et leurs traductions vers le russe (von Böhtlingk, 1849-1851; Khudyakov, 1890; Yastremsky, 1929; Pekarsky, 1934; Popov, 1936; Ergis, 1947; Poukhov, etc.). La première traduction scientifique d'olonkho en deux langues, yakoute et russe, a été publiée en 1947, c'était l'olonkho de Konstantin Orosin «Niourgoun Bootour l'Impétueux» traduit et expliqué par Georguiy Ergis. Cet ouvrage a ouvert la tradition de publication d'olonkho en deux langues.

L'intérêt scientifique pour les épopées héroïques a fait naître la série «Les monuments du folklore des peuples de la Sibérie et de l'Extrême Orient » de l'édition « Nauka » (à partir de 1990), chaque ouvrage présente deux textes en yakoute et russe et contient un disque vinyl avec l'enregistrement de la voix de conteur.

La traduction du texte d'olonkho de 36000 lignes vers le russe a vu le jour en 1975, elle appartient à Vladimir Derjavin qui a traduit le texte "Niourgoun Bootour l'Impétueux » de Platon Oyounsky, écrivain, fondateur de la littérature yakoute. Cette version volumineuse a connu un grand succès et a été ensuite traduite vers les langues étrangères : la traduction vers le slovaque par Miloš Krno en 1984 éditée à Bratislava et la traduction vers l'anglais éditée à Londres en 2014, réalisée par une équipe de traducteurs de l'Université fédérale du Nord-Est sous la direction d'Alina Nakhodkina.

La première traduction d'olonkho vers le français a paru en 1990, c'est la traduction d'olonkho de Konstantin Orosin qui a été réalisée par Yankel Karro et Lina Sabaraïkina, elle s'appelle «Niourgoun: le Yakoute, guérrier célèste. Les guerriers célestes du pays yakoute- saxa ». Le second ouvrage d'olonkho en français a vu le 
jour en 2012, c'est l'olonkho de Piotr Ogotoev «Eles Bootour » qui a été traduit par Valentina Chapochnikova.

La traduction d'une épopée héroïque est une affaire laborieuse qui peut prendre plusieurs années de travail méticuleux. Les traducteurs essaient de garder la sémantique, la stylistique, mais aussi la sonorité, la mélodie de la langue d'olonkho avec les moyens de la langue réceptrice.

\section{Problèmes de traduction d'olonkho : les realia}

Le problème du lien entre la langue et la culture a toujours représenté un grand intérêt pour la théorie et la pratique de la traduction littéraire, car le transfert et la préservation de la spécificité nationale et culturelle de l'œuvre est toujours une tâche assez difficile pour le traducteur. Il ne s'agit pas seulement d'une traduction des réalia mais aussi des mots ordinaires, qui à première vue, n'ont pas un caractère national spécifique.

Il arrive souvent que, lorsque les lecteurs perçoivent une traduction, "les informations culturelles contenues dans le texte original soient partiellement remplacées par de nouvelles informations dont la quantité ne s'avère pas toujours être égale à celle d'informations reçues en traduction"(Miculina, 1977).

Leur comparaison implique la comparaison de cultures - celle de la langue source et celle de la langue de traduction. Cet article a pour but d'examiner la signification des mots de la langue yakoute en tant que source, le russe et le français - en tant que langues de traduction et en même temps des phénomènes des cultures yakoute, russe et française. Des exemples sont tirés de l'épopée yakoute (olonkho) «Niourgoun Bootour le Yakoute Guerrier Céleste» et de ses traductions en russe (C. Ergis) et en français (J. Karro).

On sait que la tradition de la construction a ses particularités dans les cultures différentes: le balagane yakoute, l'izba russe, la maison à pignon française, le Fachwerkhaus allemand ont des caractéristiques spécifiques.

Lorsque l'œuvre traite des réalia de la vie matérielle, propres à un peuple ou à un pays, leur transfert constitue une difficulté particulière.

Cela est dû au fait que "par la fréquence d'utilisation, par le rôle dans le langage ou par leur nature quotidienne, les mots qui servent de nom à de telles réalia n'ont pas de coloration terminologique; ils ne contrastent pas avec le contexte le plus ordinaire de la langue source, ils n'y sont pas stylistiquement distingués, étant habituel pour la langue source " (Fedorov, 2002).

Si on compare les significations de mots similaires en yakoute, russe et surtout français, nous pouvons constater que les volumes d'informations culturelles sont très différents. De telles divergences ne sont pas surprenantes en matière d'information dont l'exhaustivité du transfert en traduction dépend du degré de familiarité des lecteurs avec ce phénomène culturel.

Ce phénomène peut être suivi par l'exemple du transfert de réalia concernant la distribution spatiale dans l'habitation des Yakoutes.

Dans la demeure yakoute, on a suivi le principe non seulement économique et fonctionnel mais aussi celui de social: le sud (à droite) appartenait aux hommes de la XLinguae, Volume 12 Issue 1XL, January 2019, ISSN 1337-8384, eISSN 2453-711X 
famille et aux invités d'honneur, la partie nord (à gauche) appartenait aux femmes et etait séparée de l'espace principal par une cloison. Cette partie fermée de la maison s'appelait khappakhtchy.

Un ethnographe célèbre V.L. Serochevsky a écrit: «Les filles et les femmes yakoutes ont vécu une vie très isolée par rapport aux hommes. Leur royaume est la maison et il ne reste que la moitié, moitié où ils se sont cachés lorsqu'un étranger est apparu et d'où ils ont regardé à travers les fentes de l'épaisse cloison » (Serochevsky, 1993).

Le Dictionnaire de la langue yakoute E.K. Pekarsky donne les significations au mot khappakhtchy:

1) un mur séparant (du côté nord du balagane) un lieu de couchage;

2) un lit pour une fille;

3) un cagibi;

4) un écran portable en écorce de bouleau ou une cloison coulissante en cuir près du foyer (Pekarsky, 1958).

Au début du chapitre 3, intitulé "Niourgoun devient un bootour", il s'agit de l'enfance de Niourgoun et de sa sœur cadette. En particulier, seules 4 lignes lui sont consacrées, à partir desquelles il est evident que la jeune fille menait une vie isolée, "sans se montrer au ciel ensoleillé, sans regarder aux nuages ...".

La signification fondamentale de l'expression consiste en ce qu'elle restait à la maison et faisait le ménage, tandis que son frère s'en allait pour toute la journée dans la forêt, en chassant.

"Кыьс күннээххэ көстүбүнэ, былыттаахха быгымына, халлаан диэки хайыһымына олорор кыыс буолла" (Orosine, 1947).

"Девучка жсила себе (в чулане), не показываясь солнечному небу, не выглядывая в облачную погоду, не оборачиваясь лицом на чистое небо" (Orosine, 1947).

"Elle vivait seule dans son réduit, ne visionnait pas son visage au soleil solaire, elle ne jetait pas un oeil aux nuages, ne tournait pas son visage vers le ciel pur " (Karro, 1994).

La langue source ne mentionne pas le lieu dans la demeure où habitait la jeune fille et ne donne aucune explication détaillée en distribution interieure de l'habitation, car cette information est déjà connue au lecteur yakoute. En version russe, le traducteur entre parenthèses indique déjà que la jeune fille vivait dans un tchoulane. Pour une personne possedée les connaissances de la culture russe, un tchoulane. est un petit réduit, non destiné au couchage où on stocke généralement divers objets non désirés, des objets d'hiver ou de la nourriture. Ce mot « le tchoulane» ne dit rien au lecteur sur l'organisation sociale de la maison.

Ainsi, en russe, le mot "чулан" n'a qu'une seule signification - il s'agit d'un réduit dans un bâtiment, servant généralement de débarras (Ojegove, 1997).

Dans la phrase française, le mot reduit n'a plus de parenthèses:

" Elle vivait seule dans son réduit ...".

Dans le Dictionnaire Petit Rober, le mot reduit est défini comme:

1) une petite pièce rétirée;

2) local exigu, généralement sombre et pauvre;

3) recoin (niche)dans une pièce (Petit Robert, 1997).

C'est le premier sens (la petite pièce rétirée) du lexème un réduit qui correspond au contenu de cette phrase, car il s'agit d'une petite pièce séparée du reste par un mur de planches. 
La deuxième signification du mot «un réduit» coïncide avec la signification du mot russe «чулан», ce qui est inacceptable en ce qui concerne le sens de la phrase. Ainsi, il s'est avéré que le traducteur français a mieux expliqué la signification de cette expression. Pour le lecteur russe, une image est complètement différente parce qu'elle est associée au mot « un réduit » en corrélation avec une seule signification de ce mot, à savoir celle d'une petite pièce sombre où on ne vit pas.

L'auteur de la traduction russe a transmis cette réalia à l'aide de l'adaptation culturelle, de sorte que l'accent ne met que sur une partie de l'information culturelle: une petite pièce rétirée. Mais il est déjà perçu comme quelque chose de particulier à la culture russe. Probablement, dans ce cas, il serait nécessaire de recourir à la translittération (transcription) avec une explication appropriée.

Et maintenant, on examine un autre exemple.

Niourgoun et sa sœur rentrent chez eux et voient un étranger faire quelque chose dans leur maison:

"Күөх бараан түУн ийэлэрэ күөйэн, харана түҮн хаайан халхалаан тиийэн кэллэ да, дьиэлээх киһи сыbйа - баайа хааман турда да ханас диэки барда. Ол баран ханырБастаах хаппахтчы иһиттэн сылгы түөрт ойобоһун аранас туос сандалы далбарыгар татта да, олуй - молуй быһаБын сулбу тардан ылла да, соботобун астаан баран аһаан имиппитинэн барда " (Orosine, 1947).

"Синетемная ночь наступает, черная ночь надвигается, хозяйничающий человек тихо встал, из темного чулана с дверьми и звонками вытащил 4 конских ребра и поставил на желтый берестяной стол" (Orosine, 1947).

"La nuit bleue - sombre avance, la leiténére s'approche le seigneur des lieux lentement se leve du reduit à provision muni de portes à sonnailles. Il traîne quatre côtes de cheval qu'il pose sur une table en ecorce de bouleau jaune" (Karro, 1994).

Dans ce cas, le mot khappakhtchy apparaît dans le texte, mais nous parlons déjà d'une autre pièce.

Comme on a déjà mentionné ci-dessus en russe, le mot "чулан" n'a qu'une seule signification: une loge, qui sert de débarras. Mais il convient de noter que le mot khappaktchi a une longue tradition de traduction comme чулан. Dans l'étude ethnographique de V. Seroshevsky "Yakouty", paru en 1896, ce mot est translittéré et transmis sous forme de khappakhtchi (Serochevsky, 1993). Dans ce cas, la traduction du mot khappakhtchy en tant que чулан est plus précise.

Le traducteur français apporte des précisions sur le sens du mot réduit en ajoutant une expression à provision, qui signifie un lieu aménagé pour y conserver la provision. Il s'avère que nous parlons déjà d'une autre pièce.

Dans cet exemple, nous voyons que le réduit a une fonction différente, on y stocke de la provision, ce qui signifie que cette pièce n'est pas destinée à accueillir une fille, bien qu'il y ait une porte avec des clochettes (Serochevsky, 1993).

Lorsqu'on compare deux traductions, on peut conclure qu'en traduction russe, une pièce - un tchoulan - sert à la fois de chambre pour une fille et de débarras pour les provisions; en français, deux salles différentes sont conçues - reduit et reduit à provision.

Dans la traduction russe, il y a un autre élément qui "russifie" un peu la demeure yakoute. C'est la présence de la porte du réduit, qui n'est pas mentionnée dans la langue source. Si on parle d'une pièce destinée à une fille, dans ce cas, la porte a été remplacée par une sonnerie suspendue au-dessus de l'entrée. Parfois c'était un morceau en tissu grossier ou en peau qui servait aussi la porte. Mais en tant que tel,

XLinguae, Volume 12 Issue 1XL, January 2019, ISSN 1337-8384, eISSN 2453-711X 
il n'y avait qu'une porte dans la pièce. L'absence d'espaces fermés dans l'habitation des Yakoutes s'explique par le fait qu'en hiver, lorsque la température atteignait souvent 60 degrés au-dessous de zéro, il était nécessaire de réchauffer constamment la pièce. Cela pourrait être fait uniquement dans une pièce qui ne comportait pas beaucoup de partitions et, bien sûr, des portes supplémentaires.

Très souvent les notes du traducteur ne donnent pas toute l'information culturelle. Ainsi, nous avons une explication suivante du mot balagan dans une note: balagan demeure en bois, de forme piramidale. Le commentaire explique le mot, mais néanmoins, il ne révèle pas une partie importante de cette information culturelle: le logement est construit de rondins verticaux et est destiné à l'habitation permanente. Nous faisons l'attention à ces informations car, dans le même ouvrage faisant référence à ce lexème, on peut déjà trouver une explication dans le texte.

“Ол түһэн баран, үс төгүл күн диэки өттүнэн холоруктаатабыма саар булгунньах са5а айданнах балақан буола түстэ...” (Orosine, 1947).

"И вот, спустившись (Мелсют Беге), едва трижды повертелся по ходу солнца, как появился широкий дом - балаган величиной с круглый большой холм" (Orosine, 1947).

A peine descendu, Mëlsjut Bëgë tourna trois fois selon la marche du soleil et une maison d'été (balagan) apparut telle une colline ronde" (Karro, 1994).

Qu'est-ce qui a motivé le choix du traducteur dans ce cas?

Dans le Dictionnaire russe, le mot balagan est défini comme une construction temporaire en planches pour divers besoins (pour le commerce, le logement, un entrepôt, etc.) (Ojegove, 1997). Le sème de "fragilité", "décroissance" est présent dans le sens de ce mot.

Si on parle de l'étymologie de ce mot, il vient du balahanä le mot persan et signifiait un balcon. Selon V.L. Serochevsky, le mot «balagane»a été introduit en Yakoutie par les Russes au XVIIe siècle. Perdant peu à peu son sens initial, il commence à désigner le type de bâtiment destiné à l'habitation permanente. Le traducteur français, utilisant le sens du mot russe «balagan» et ne voyant pas la spécificité nationale du mot yakoute balagan, a suggéré qu'il s'agissait d'un bâtiment provisoire, à la suite duquel la traduction la vaste maison d'été est parue.

\section{Problèmes de traduction d'olonkho : les connotations culturelles}

Pour illustrer les procédés de traduction des connotations culturelles ethniques nous avons choisi un texte de l'épopée yakoute «Niourgoun le Yakoute, guerrier céleste» traduit en français par Yankel Karro. Une des formules les plus répandues dans les textes épiques est une comparaison avec le mot — kytalyk\| (la grue). On compare à — kytalyk\| les jeunes filles, les jeunes gens, les personnages principaux féminins.

Dans la langue yakoute, cette comparaison est devenue une formule figée, une des composantes propres à la structure et au genre du texte épique. C'est un vrai concept de la culture matérielle et spirituelle qui remonte aux époques archaïques des ancêtres yakoutes qui étaient poussés des régions des monts Sayan vers le Grand Nord sibérien par l'expansion mongole-bouriate-oïrate, au moment des conquêtes de Genguis-Khan. Dans la version française de ce texte épique le traducteur Y.Karro utilise quatre termes différents comme équivalent du mot kytalyk: la grue, la cigogne, le héron et la 
forme translittérée «kytalyk ». Pour essayer d'expliquer le choix du traducteur nous nous sommes adressés aux dictionnaires et encyclopédies des symboles.

Le dictionnaire Hachette de notre temps pour le mot «la grue» fixe deux significations courantes: 1) oiseau migrateur de grande taille; 2) pop. prostituée, fille légère. Une encyclopédie des symboles nous montre que, dans la culture occidentale, cet oiseau est associé à l'idée de sottise, de maladresse. Dans la culture orientale, la grue symbolise la longévité, la fidélité, le respect envers ses parents. Le mot « la cigogne » signifie aussi un grand oiseau échassier, migrateur. Mais ses connotations sont différentes de celles de la grue: en Egypte la cigogne symbolise la chance, la résurrection, la nouvelle vie; dans les pays orientaux - la longévité, la fidélite, mais aussi la contemplation, la méditation et la vigilance.

Le héron dans les cultures européennes et africaines est associé avec la curiosité extrême; dans le christianisme il symbolise la pureté, la sobriété et la discrétion.

Dans la traduction française on rencontre ces trois mots et, en plus, la forme translittérée du mot yakoute «kytalyk ». Dans la culture yakoute la grue blanche signifie non seulement le symbole de beauté, de pureté, mais tout un ensemble d'idées et de notions qui expriment le bonheur, la vie heureuse, le sens de vie, tout un univers comme un écoumène béni. Le traducteur Yankel Karro utilise quatre termes différents comme les solutions possibles pour transposer en français le mot yakoute « kytalyk »: la grue, la cigogne, le héron et la forme translittérée « kytalyk » qui se diffèrenr non seulement par leurs significations dénotatives, mais aussi connotatives. Pour la première fois, la transposition du mot $\|$ kytakyk se trouve dans le contexte où les vieux parents du futur héros Niourgoun, obligés d'envoyer leurs enfants (leur fils et leur fille) du ciel où ils vivent dans le Pays du Milieu pour sauvegarder les gens de la tribuedes Aiy (les peuples yakoutes), s'adressent à leurs domestiques: Plus vite, allons, vous les neufs gaillards pareils aux grues robustes, vous les neuf filles pareilles aux grues graciles, parez-les (nos enfants) tel le vert bosquet. Yankel Karro a fait quelques substitutions lexicales. Dans le texte original, il y a deux termes distincts pour le mot - la gruell: on compare les jeunes hommes à des grues grises (en yakoute tourouya), et les jeunes filles aux femelles des grues blanches (les femelles des kytalyks). Yankel Karro a utilisé pour les deux mots yakoutes un mot -la gruell. Il a omis le mot — femellell, probablement, pour éviter une ambiguïté non désirable qui vient de l'usage familier du mot — gruell. A la place du mot - femellel, Y.Karro emploie le mot — gracilell qui est très loin de l'idée de la femelle mais compotre une idée de beauté, de jeunesse. Cette idée s'harmonise bien avec le sens symbolique de cet oiseau: la sottise, la légèreté. Dans ce cas-là, le sens penche vers l'idée de légèreté, de jeunesse. Outre cela, dans la version française, les pertes et les modulations lexicales sont compensées par un rythme et une homophonie créés à l'aide d'une allitération de $-\mathrm{r} \|,-$ grll, ce qui augmente les caractéristiques esthétiques du texte français. Une telle stratégie sert à assimiler l'oeuvre traduite pour la culture-cible. La deuxième fois, la traduction du mot - la gruell se rencontre dans le même fragment de texte: la vieille mère des enfants envoyés en mission dit à ses servantes: Mes huit filles, vous mes filles, vous mes servantes, mes cigognes, mes kytalyk, venez avec moi cueillir mon petit enfant, ornons-la comme la terre des fleurs, parons-la, légère et belle. Le traducteur refuse d'utiliser le mot — la gruell en le substituant par le mot - la cigognell et la forme translittérée - kytalyk\| pour laquelle Karro donne l'explication suivante:loiseau blanc très gracieux semblable à la gruell. A notre avis le choix du 
terme — cigognell peut être justifié par deux raisons: premièrement, ce sont les paroles d'une vieille femme vénérable, mère du futur héros, adressées à ses servantes; la répétition multiple du pronon possessif mes accentue l'idée de possession, de rapprochement, ce qui met la mère et ses servantes sur le même pied d'égalité, par conséquent, le vecteur de l'intention pragmatique de l'auteur du texte-cible va vers une appréciation positive de tous les participants de cet acte de parole. Deuxièmement, le sens symbolique propre à la cigogne dans la culture occidentale est - la chancell, - le bonheur\| qui oriente aussi le traducteur vers ce choix pour anticiper la gloire fameuse du héros principal Niourgoun Botour. L'exemple suivant illustre une traduction tout à fait différente du terme — gruel par le terme - héron\| dans un énoncé où l'on compare la soeur du héros principal qui s'appelle Aytaly Kuo: Elle était si belle qu'on aurait dit la femelle du héroll. Cette phrase a gardé la syntaxe et le lexique de la phrase yakoute, sauf la substitution du mot — gruell par le mot - héronll. On peut supposer qu'il y a des facteurs qui ont influancé le choix du mot —héronll. On dirait que l'auteur différencie entre le personnage du premier rang (la soeur du héros principal) des autres (les domestiques). Pour établir cet indice —d'inégalitél, Y. Karro consciemment / ou inconsciemment introduit un nouveau mot qui signifie aussi un oiseau échassier, mais qui se caractérise par des qualités symboliques tout à fait différentes: le héron symbolise la discrétion, la modération, ces qualités s'harmonisent bien avec les significations de base du mot — femellell. La dernière fois le mot — la gruell se rencontre dans le contexte où il s'agit de l'apparition miraculeuse d'une chamanesse sous forme de grue: à droite du Botour, se posa l'oiseau kytalyk. Seule la forme translittérée - kytakyk\| peut transmettre une idée de magie, de miracle liée à une chamanesse. Donc, nous avons vu que le mot yakoute - kytalykll qui présente un concept culturel dans le texte est traduit en français par des termes différents; non seulement du point de vue des dénotations, mais aussi des connotations, qui sont chargées de significations symboliques différentes. Celle solution du traducteur Y.Karro peut être expliquée par deux raisons au moins: 1) le mot - la gruellqui est l'équivalent lexical du mot yakoute — kytalyk\| dans la langue française usuelle, a des connotations négatives dans le langage populaire, par conséquent, il existe un obstacle lexical pour l'emploi libre de ce terme; 2) le concept de la culture yakoute à la base de kytalyk n'existe pas dans la culture française, les comparaisons avec ce mot ne sont pour lui que des procédés stylistiques embellissant le texte épique. Mais étant parfaitement conscient de spécificités de la culture spirituelle yakoute, il crée un nouveau concept sur base de la - gruell en détachant les mailles de ce filet de la surface du champs conceptuel. Chaque maille est assez autonome du point de vue sémantique, dénotatif et connotatif. Par contre, le concept yakoute $\|$ kytalyk\| a une structure homogène à grande capacité sémantique et connotative, comme les facettes d'une pierre taillée font des rayons indissociables qui reflètent une image unique.

\section{Problèmes de traduction d'olonkho : l'aspect du genre}

La compréhension du texte d'origine est aggravée par certains circonstances linguistiques et extralinguistiques. Ce sont les savoirs-faire de la langue, de l'objet d'enoncé, des réalitées locales et objectives dans le discours d'origine.

La traduction suppose la pénétration dans la forme du texte original, qui a son contenu et en revele l'idée de l'auteur. Ainsi il est important de respecter les spécificités d'enoncé dans la langue. 
En concernant le thésaurus subjectif de l'auteur, dans l'étude du texte de la traduction, nous repérons la necessité du respect de l'appartenance d'auteur non seulement à la culture, mais aussi au sexe social. "Les relations de genre sont enregistrées dans la langue sous la forme de stéréotypes culturels et conditionnés, imprimant une empreinte sur le comportement, y compris la parole, la personnalité et les processus de sa socialisation linguistique» (Kirilina 1999, 90). À cet égard, il est important de prendre en compte les caractéristiques des stéréotypes de masculinité et de féminité adoptés dans la culture étudiée et reflétés dans la langue. L'activité subjective de l'auteur se déroule selon les règles objectives de la composition du texte dans le groupe linguistique. Ces règles conservent, sous chaque forme de texte, certaines règles propres à sa structure logique et grammaticale, et codent des informations pragmatiques (pertinentes), en se concentrant sur un lecteur et une compréhension adéquate» (Danilova 2001, 16).

Le texte artistique est un objet de traduction spécifique. Fonctionnant comme une œuvre esthétique, le texte artistique est destiné non seulement à transmettre des informations, mais à créer un certain effet esthétique, à produire un effet émotionnel sur le destinataire, à provoquer dans l'esprit du lecteur des images émotionnelles et colorées, des associations motivées, etc. (Danilova 2001, 40). Dans le processus de traduction, on révèle des éléments qui ne sont pas formalisables et qui font «des unités de microstructure artistique, à l'aide desquelles les lecteurs ont certains états mentaux discrets» (Sorokin 1985, 138). En d'autres termes, les textes de la traduction ne peuvent pas être compris par les lecteurs sans perdre une partie du sens.

L'acte de traduction est un acte créatif, bien que secondaire, subordonné. Ce qui se produit à la suite, il y a une nouvelle œuvre (Bouroukina 2000, 105).

Après Bouroukina O.A., nous affirmons que «toute œuvre originale n'est perçue par les locuteurs natifs que si toutes les lois sont respectées, y compris le facteur de genre, souvent plus important que de nombreux autres aspects stylistiques et grammaticaux (Bouroukina 2000, 106).

Le sujet de l'étude est une étude comparative de genre du texte de l'épopée yakoute "Nyurgun Bootur le guerrier célèste" et sa traduction en français, réalisée par Yankel Carro. La langue russe était la langue intermédiaire dans la mise en œuvre de cette traduction par Guermoguenov G. U - Ergis.

"L'image mentale d'un objet transféré d'une culture à l'autre comporte toujours des éléments de spécificité culturelle, car les nouvelles connaissances sur la culture étrangère sont conçues à partir des connaissances du passé du sujet d'analyse" (Tarassov 1996, 19). Ainsi, le traducteur-transporteur de la culture occidentale dans certains cas a tendance à idéaliser l'image masculine, offrant à l'homme un rôle prédominant. Carreau Y. a fait le remplacement lexical et en même-temps le changement du déroulement sémantique du contexte, en utilisant au lieu du mot «la vieille» le mot «le vieillard». Dans le texte de l'origine et dans le texte russe la parole de gloire et de fierté pour son fils appartient à la mère. Alors que dans la traduction en français, au lieu de la mère, c'est le père qui prononce de gloire et de fierté pour son fils. Les parents âgés des enfants, s'étant désolés, obéissent à la volonté des divinités supérieures et commencent à préparer les enfants au déménagement. La mère, après avoir vu l'armure de combat et l'équipement de son fils, crie: «Да, действительно 
правда, наш сын был создан определением Одун Бииса, был рожден указом Дьылга Тойона. Ныне я этому радуюсь и ликую!». La traduction en français est: «Ayant vu armes et armures de son fils, le Bôtour Niourgoun l'Audacieux, voici ce que dit le vieillard: «Oui, verité des verités, notre fils vint par la decision d'Odoun Bîs, c'est l'ordre de D'ylga Tojon qu'il l'a fait naître, aujourd'hui je me rejouis, j'exulte!». Il semble que le traducteur remplace délibérément le mot "la vieille" par le contraire "le vieillard". Dans la culture turque yakoute, la mère a une position hiérarchique plus élevée et une plus grande importance socioculturelle que dans la culture occidentale. En outre, le texte de la traduction perd l'inquiétude de la mère pour la vie et le sort de son fils, qui va quitter sa maison et devenir un défenseur. Cela provoque un nouveau sens - l'instruction du père au fils. Le traducteur a complètement remplacé l'unité lexicale et, en même temps, le déroulement sémantique du contexte. Ainsi, le traducteur offre le rôle le plus important au père, et non à la mère, lui donnant le droit d'exprimer la fierté pour le fils.

Dans la culture yakoute, l'image de la mère est comparée au soleil-Kune Kubej Khotoune Ije, ce qui signifie littéralement la mère honorable comme le soleil, où "kune" signifie le soleil, "kubej" (en liaison avec khotune) - honorable, importante, vénérable, éminente, calme, gracieuse, légère et ije - une mère. À notre avis, dans la traduction en russe l'appel du héros à la mère “Солнцеликая матушка мать моя!" qui signifit "ma mère au visage du soleil", au lieu de dire «Күн күбэй хотун ийэм!» en yakoute, perd la valeur du respect de la mère qui éprouve et exprime le jeune homme, et acquiert une teinte plus émotionnelle. Le traducteur a essayé de faire la communication la plus proche possible du texte en russe. Les textes de l'origine et de la traduction doivent être équivalents dans leur valeur communicative, ayant un effet communicatif égal, c'est-à-dire provoquer dans l'esprit du destinataire des images et des associations similaires à celles que l'auteur du texte de l'origine voudrait informer. Selon A. Popovitch, "pour la traduction artistique, il est important que les faits linguistiques et thématiques soient réalisés dans la construction stylistique du texte, et le point clé dans la théorie de la traduction artistique devrait être considéré comme le concept de l'information esthétique invariante". L'évolution des faits et du contenu thématique est due à des différences culturelles. "La meilleure façon de résoudre le problème de l'équivalence traduit est l'approche fonctionnelle et stylistique, qui vise à transmettre de manière adéquate le caractère stylistique de texte de l'origine dans d'autres systèmes linguistiques et culturels" (Danilova 2001, 42).

Ainsi, dans cette phrase le traducteur du yakoute en russe a essayé de provoquer pour les destinataires russophones des images et des associations similaires ou proches de la culture russe, en tenant compte la nature stylistique. L'image de la mère acquiert une teinte émotionnelle "ma mère, mon aimée au visage de soleil"-“солнцеликая матушка", c'est-à-dire la mère est claire, chaude comme le soleil. "L'image de la mère dans la conscience de langue des russes est associée à la chaleur, à la gentillesse, aux soins" (Kirilina 1999). L'information conceptuelle et culturelle du texte yakoute est perdue dans les textes de traduction en raison de "l'information esthétique".

La mère dans la culture yakoute est bien appréciée. Sans aucun doute, elle est légère, gentille, chaude comme le soleil. Cependant, à notre avis, la comparaison de la mère avec le soleil remonte des anciennes vues de yakoutes. Les anciens yakoutes adoraient le soleil. «Le soleil est la source de toute vie sur la terre et de l'énergie...Donc, "les anciens yakoutes rencontraient le lever du soleil pendant Yssyakh, la fête nationale chez les yakoutes avec l'arrivée de l'été" (Syrdyk, 2001, 78). il est nécessaire de noter 
que cette tradition vit aujourd'hui. Selon les représentations des yakoutes, "le soleil symbolise la divinité supérieure Iejekhsit (déesse ancestrale), qui a une relation étroite avec le soleil" (Ibid., 88). Selon Ojuunsky P. A., la divinité et la foi des anciens yakoutes remonte du culte de la femme (Ojuunsky, 1962, 43). Ainsi, dans la culture yakoute, l'image de la mère par rapport au soleil a une signification sacrée.

Le discours de la bogatyrka (de l'amazone) dans le texte de la traduction française présente un bon exemple qui respecte des spécificités de l'aspect de genre. Un bogatyr (le preux), le héros est venu s'épouser à une bogatyrka, la héroine. Mais, en défendant son droit à l'existence indépendante, une femme essaie de sembler aux yeux de l'homme non seulement forte et intrépide, mais elle se met en place hiérarchiquement plus haut que l' homme. Ainsi, elle dit: “Ты пришел к высокому имени, ты приехал к доброй славе такой женщины как я? Как думаешь, ты победить такого человека как я?» qui est traduit «Tu es venu vers le grand nom, tu as pris la route vers la noble gloire d'une femme telle que moi.. comment penses- tu vaincre un être comme moi?». Le traducteur a transmit le mot "homme" par le mot "être" qui désigne une créature, une personne en général, sans indiquer son appartenance sexuelle. C'est une créature sur un homme et une femme. Il est probable que le choix d'un interprète a été influencé par tel fait que l'équivalent français de "l'homme" désigne une personne de sexe masculin. Dans ce contexte, ce mot "l'homme" ne convient pas du tout. L'utilisation du mot "un être" transmet l'image d'une femme forte qui se considère plus supérieure que l'homme. C'est presque un surhumain, pas un homme ou une femme.

Dans la scène de la lutte de la femme-héroine et de l'homme-héros, à notre avis, la décription du comportement de la héroine pendant le combat attire notre attention. La proposition «из корней волос женщины выступил белый пот... своими прекрасными белыми пальцами смахнула пот» est traduit en français «une sueur blanche s'échappait de la racine de ses cheveux, et de ses doigts magnifiques et forts elle fit ruisseler le sueur de deux côtés». Le traducteur remplace l'adjectif “белые/blancs" par “сильные/forts". Le remplacement des adjectifs en raison de "forts" est plus approprié dans le contexte, c'est - à-dire que les doigts d'une femme forte devraient être forts. Toutefois, le traducteur ne tient pas compte de la parité de genre. Ainsi, dans le texte russe, la féminité de la bogatyrka (amazone) se manifeste clairement. Bien qu'elle est forte ou courageuse, elle ne perd pas sa féminité, elle balaie la sueur avec ses beaux doigts blancs. Ainsi, dans le texte de la traduction en français, le traducteur ne tient pas compte de la qualité de la féminité de l'amazone, qui est transmise dans la version russe.

L'aspect du genre est un problème de traduction assez complexe que le traducteur doit obligatoirement prendre en compte pour créer une œuvre littéraire équivalente et égale en valeur en contenu dans la langue de traduction.

\section{Conclusion}

Les difficultés du premier ordre qui font des obstacles pour le traducteur sont les realia et les notions chargées des connotations spécifiques propres à la culture du texte original. Les diverses possibilités de traduction et de transferts des particularités culturelles liées avec les notions et concepts ethniques sont montrées dans l'article : les procédés de traduction visant à conserver l'image spécifique telle que la 
translittération, et d'autre part, les procédés qui «naturalisent » les objets de culture à l'aide de l'adaptation des concepts étrangers pour la culture réceptrice.

Nous pouvons faire les conclusions suivantes:

Le vocabulaire de la culture nationale se manifeste à différents niveaux, ce qui entraîne de grandes disparités entre les mots des trois langues comparées. Les tentatives de préservation de la culture nationale de l'original conduisent souvent à la formation de violations sémantiques de profondeurs différentes, causées par l'influence de la culture traductrice et le désir d'adapter des éléments d'une autre culture. La saveur culturelle nationale de l'œuvre de folklore est effacée en raison de l'utilisation la plus fréquente de méthodes de transmission de réalia telles que la traduction approximative et la généralisation.

Les exemples cités dans l'article n'indiquent en aucun cas que le transfert de spécificités culturelles nationales vers une autre langue dans la traduction littéraire est impossible. Au contraire, ce problème a récemment attiré beaucoup d'attention des linguistes. De nombreuses études de traduction contribuent à la fois à décrire le caractère unique et la diversité des langues et des cultures et à développer davantage la communication interlinguistique et interculturelle.

\section{Bibliographic references}

BOUROUKINA O.A. 2000. Gendernyj aspect perevoda [L'aspect de genre de la traduction]. In: Gender kak intriga poznanija. Moscou, p. 99-107. ISBN 5-7380-01311

GUERMOGUENOV G.U. Ergis. 1947. N'urgun Bootur Stremitelnyj [Niourgoun Bootur Impétuex]. Yakutsk.

DANILOVA E.V. 2001. Psycholingvistitcheskij analiz vosprijatija khoudogestvennogo teksta $\mathrm{v}$ raznykh kul'turakh [L'analyse psycholinguistique de perception du texte artistique chez les différentes civilisations]: la thèse de docteur en philologie. Moscovskij gos. lingvistitcheskij universitet, Moscou, 204 p.

IVANOV V.N. 2016. Istoriki o proiskhojdenii yakutskogo geroicheskogo eposa [Les historiens sur la génèse de l'épopée héroïque yakoute]. In: Vestnik Tomskogo gosudarstvennogo universiteta, n. 404, pp.89-95. DOI: 10.17223/15617793/404/13

KARRO Y. 1997. Niourgoun: le Yakoute, guérrier célèste. Les guérriers célèstes du pays yakoute- saxa. Paris. Gallimard, Torino.

KIRILINA A.V. 1999. Gender: lingvistitcheskij aspect [Genre: l'aspect linguistique]. Moscou, $155 \mathrm{p}$.

KHUDYAKOV I.A. Verkhoyanskiy sbornik. Yakutskiye skazki, pesni, zagadki i poslovitsy, a takzhe russkiye skazki i pesni, zapisannyye v Verkhoyanskom okruge [Verkhoyansk collection. Yakut fairy tales, songs, riddles and proverbs, as well as Russian fairy tales and songs recorded in the Verkhoyansk district]. Irkutsk, 1890. (In Russ.).

MOUNIN G. 1980. Les problèmes théoriques de la traduction, Paris, Gallimard.

OJUUNSKY P.A. 1962. Yakutskaja skazka (olonkho), eje sujet I soderjanije [La conte yakoute (olonkho), son sujet et contenu]. In: Proivedenije, Volume 7, Yakutsk. 224 p.

POUKHOV, I.V. Olonkho - drevniï epos yakoutov [L'olonkho - l'ancienne épopée des Yakoutes].

SOROKIN Ju. A. 1985. Psycholingvistitcheskije aspect izutchenija teksta [L'aspect psycholinguistique]. Moscou. $168 \mathrm{p}$.

SPEKTOR, L.A. Natsionalno-koultournaya spetsifika i perevod [Subtilités culturelles nationales et traduction].Vestnik UFNE, v.2. Yakoutsk, 2005.- P. 26-29. ISSN 22225404 
SPEKTOR, L.A. Smyslovye ochibky pri peredatche etnografitcheskih realii [Fauxsens dans le transfert des réalia ethnographiques] Obchtchestvennye naouki. Moscou, n. 6-1, 2016. P.172-179. ISSN 2079-5505

TARASSOV E.F. 1996. Mejculturnoje obshchenije - novaja ontologija jazykovogo soznanija [La communication intercuturelle - nouvelle ontologie de conscience linguistique] In Etnoculturnaja spetsifika jazykovogo soznanija. Moscou. pp. 7-22.

TARASSOV E.F. 1998. K postroenii teorii mejkulturnogo obshenia/ [De la construction de la theorie de la communication interculturelle] In Yazykovoe soznanie: formirovanie i funksionirovanie, Moscou, pp. 30-34.

VOGELEER Sv. 1995. L'interprétation du texte et la traduction, Louvain-la-Neuve, Peeter.

VON BOHTLINGK, Otto. Uber die Sprache der Jakuten («O языке якутов») (в 3 томах, Санкт-Петербург, 1849-1851);

YADRIKHINSKYY-BED'EELE P.P. Devushka-bogatyr' Dzhyrybyna Dzhyrylyatta [The hero-girl Dzhyrybyna Dzhyrylyatta]. Yakutsk, 2011. (In Russ.).

YASTREMSKY S.V. Obraztsy narodnoy literatury yakutov [Samples of folk literature of the Yakuts]. Leningrad, 1929. (In Russ.).

DICTIONNAIRE DES SYMBOLES, Paris, 1982. Encyclopédie des symboles, Torino, 1997.

YAKUTSKIY GEROICHESKIY EPOS "KYYS DEBILIYE" [Yakut heroic epic "Kyys Debiliye"]. Novosibirsk, 1993. (In Russ.). 12. Yakutskiy geroicheskiy epos "Moguchiy Er Sogotokh" [Yakut heroic epic "Mighty Er Sogotokh"]. Novosibirsk, 1996. (In Russ.).

YAKUTSKIY FOLKLOR. Teksty i perevody A.A. Popova [Yakut folklore. Texts and translations by A.A. Popov]. Moscow, Leningrad, 1936. (In Russ.).

Words: 7447

Characters: 48233 (26,80 standard pages)

Associate Professor Liudmila S. Zamorshchikova

Associate Professor Marianna V. Samsonova

Associate Professor Lilya A. Spector

Associate Professor Irena S. Khokholova

North-Eastern Federal University, PhD,

Institute of Modern Languages and Regional Studies,

Department of French,

58, Belinskogo Str., 677007 Yakutsk, Sakha Republic,

Russia

1szam@mail.ru

efimarianne@mail.ru

libondarenko@mail.ru

iskhokholova@mail.ru

XLinguae, Volume 12 Issue 1XL, January 2019, ISSN 1337-8384, eISSN 2453-711X 